Pacific

Journal of

Mathematics

\title{
ON THE FINITE GENERATION OF
}

A FAMILY OF EXT MODULES

TONY J. PUTHENPURAKAL 


\title{
ON THE FINITE GENERATION OF A FAMILY OF EXT MODULES
}

\author{
TONY J. PUTHENPURAKAL
}

Dedicated to Professor L. L. Avramov on the occasion of his sixtieth birthday.

Let $(A, \mathfrak{m})$ be a local complete intersection ring. Let $M, N$ be finitely generated $A$-modules and let $I$ be an ideal in $A$. We show that

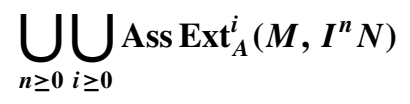

is a finite set. We also show that there exist $i_{0}, n_{0}$ such that for all $i \geq i_{0}$ and $n \geq n_{0}$ we have

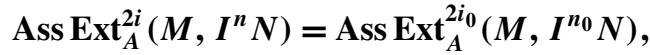

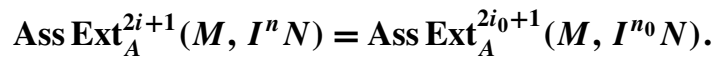

We prove analogous results for complete intersection rings which arise in algebraic geometry. We also prove that the complexity, $\operatorname{cx}\left(M, I^{n} N\right)$, is constant for all $n \gg 0$.

\section{Introduction}

Let $A$ be a Noetherian ring. Let $I$ be an ideal in $A$ and let $M$ be a finitely generated $A$-module. M. Brodmann [1979] proved that the set $\operatorname{Ass}_{A} M / I^{n} M$ is independent of $n$ for all large $n$. This result is usually deduced by proving that $\operatorname{Ass}_{A} I^{n} M / I^{n+1} M$ is independent of $n$ for all large $n$.

We state some generalizations of Brodmann's result. Fix $i \geq 0$. L. Melkersson and P. Schenzel [1993, Theorem 1] showed that

$$
\operatorname{Ass}_{A} \operatorname{Tor}_{i}^{A}\left(M, I^{n} / I^{n+1}\right) \text { and } \operatorname{Ass}_{A} \operatorname{Tor}_{i}^{A}\left(M, A / I^{n}\right)
$$

are independent of $n$ for all large $n$. By the same argument,

$$
\operatorname{Ass}_{A} \operatorname{Ext}_{A}^{i}\left(M, I^{n} / I^{n+1}\right)
$$

The work for this paper was done while the author was visiting University of Kentucky by a fellowship from Department of Science and Technology, India. The author is deeply grateful to DST for its financial support and University of Kentucky for its hospitality.

MSC2010: primary 13D07, 13H10; secondary 13A15, $13 \mathrm{~A} 02$.

Keywords: local complete intersection, asymptotic associate primes, cohomological operators. 
and, by [Katz and West 2004, 3.5],

$$
\operatorname{Ass}_{A} \operatorname{Ext}_{A}^{i}\left(M, A / I^{n}\right)
$$

are similarly independent of $n$. An example of A. Singh [2000] shows that

$$
\operatorname{Ass}_{A} \lim _{\rightarrow} \operatorname{Ext}_{A}^{i}\left(A / I^{n}, M\right) \text { need not be finite. }
$$

So in this example

$$
\bigcup_{n \geq 1} \operatorname{Ass}_{A} \operatorname{Ext}_{A}^{i}\left(A / I^{n}, M\right) \text { is not even finite. }
$$

I state some questions in this area that motivated me. They were raised respectively by W. Vasconcelos [1998, 3.5] and Melkersson and Schenzel [1993, page 936].

$$
\text { Is the set } \bigcup_{i \geq 0} \operatorname{Ass}_{A} \operatorname{Ext}_{A}^{i}(M, A) \text { finite? }
$$

$$
\text { Is the set } \bigcup_{i \geq 0} \bigcup_{n \geq 0} \operatorname{Ass}_{A} \operatorname{Tor}_{i}^{A}\left(M, A / I^{n}\right) \text { finite? }
$$

The motivation for the main result of this paper came from (1). I do not believe that the question has a positive answer in this generality, but I am unable to give a counterexample. Note that if $A$ is a Gorenstein local ring then Vasconcelos's question has, trivially, a positive answer. If we change the question a little then we may ask: If $M, D$ are two finitely generated $A$-modules,

$$
\text { is the set } \bigcup_{i \geq 0} \operatorname{Ass}_{A} \operatorname{Ext}_{A}^{i}(M, D) \text { finite? }
$$

This is not known for Gorenstein rings in general. However, if $A=Q /(f)$, where $\boldsymbol{f}=f_{1}, \ldots, f_{c}$ is a regular sequence, and if $\operatorname{projdim}_{Q} M$ is finite, then the above question has a positive answer. This can be seen by using the theory of cohomology operators over such rings. This turns $\bigoplus_{i \geq 0} \operatorname{Ext}_{A}^{i}(M, D)$ into a finitely generated module over $A\left[t_{1}, \ldots, t_{c}\right]$, where $t_{i}$ has degree 2 for each $i$.

Using Melkerson and Schenzel's question as a guidepost, I was interested to solve the the following questions: Let $(A, \mathfrak{m})$ be a local complete intersection of codimension $c$.

$$
\text { Is the set } \bigcup_{i \geq 0} \bigcup_{j \geq 0} \operatorname{Ass}_{A} \operatorname{Ext}_{A}^{i}\left(M, D / I^{j} D\right) \text { finite? }
$$

$$
\text { Is the set } \bigcup_{i \geq 0} \bigcup_{j \geq 0} \operatorname{Ass}_{A} \operatorname{Ext}_{A}^{i}\left(M, I^{j} D\right) \text { finite? }
$$

In Theorem 5.1 I prove that (b) holds. I have been unable to verify whether (a) holds. 
Let $\mathscr{R}(I)=\bigoplus_{n \geq 0} I^{n} t^{n}$ be the Rees algebra of $I$. The main result in this paper concerns finite generation of a family of Ext modules:

Theorem 1.1. Let $Q$ be a Noetherian ring with finite Krull dimension and let $\boldsymbol{f}=$ $f_{1}, \ldots f_{c}$ be a regular sequence in $Q$. Set $A=Q /(f)$. Let $M$ be a finitely generated $A$-module with projdim
finitely generated $\mathscr{R}(I)$-module. Then

$$
\mathscr{E}(N)=\bigoplus_{i \geq 0} \bigoplus_{n \geq 0} \operatorname{Ext}_{A}^{i}\left(M, N_{n}\right)
$$

is a finitely generated bigraded $\mathscr{Y}=\mathscr{R}(I)\left[t_{1}, \ldots, t_{c}\right]$-module.

Remark 1.2. See Section 2.3 for a description of $\mathscr{E}(N)$ as a $\mathscr{S}=\mathscr{R}(I)\left[t_{1}, \ldots, t_{c}\right]$ module.

An easy consequence of this result is that (b) holds (by taking $N=\bigoplus_{n \geq 0} I^{n} D$ ); see Theorem 5.2. A complete, local complete intersection ring is a quotient of a regular local ring mod a regular sequence. So in this case (b) holds from Theorem 5.2. The proof of (b) for local complete intersections in general is a little technical; see Theorem 5.1. We also prove (b) for complete intersection rings which arise in algebraic geometry; see Section 6.

We next discuss a surprising consequence of Theorem 1.1. Let $(A, \mathfrak{m})$ be a local complete intersection of codimension $c$. Let $M, N$ be two finitely generated $A$ modules. Let $\mu(X)$ denote the number of minimal generators of a finitely generated $A$-module $X$. Define

$$
\operatorname{cx}_{A}(M, N)=\inf \left\{b \in \mathbb{N} \mid \varlimsup_{n \rightarrow \infty} \frac{\mu\left(\operatorname{Ext}_{A}^{n}(M, N)\right)}{n^{b-1}}<\infty\right\} .
$$

In Section 7 we prove (see Theorem 7.1) that

$$
\operatorname{cx}_{A}\left(M, I^{j} N\right) \text { is constant for all } j \gg 0 .
$$

We now describe in brief the contents of this paper. In Section 2 we give a module structure to $\mathscr{E}(N)$ over $\mathscr{Y}$ (as in Theorem 1.1). We also discuss a few preliminaries. The local case of Theorem 1.1 is proved in Section 3 while the global case is proved in Section 4. In Section 5 we prove our results on asymptotic primes in the case of local complete intersections. In Section 6 we prove our result on asymptotic primes in complete intersection rings which arise in algebraic geometry. In Section 7 we prove $(\dagger)$.

\section{Module structure}

Let $Q$ be a Noetherian ring and let $\boldsymbol{f}=f_{1}, \ldots f_{c}$ be a regular sequence in $Q$. Set $A=Q /(\boldsymbol{f})$. Let $M$ be a finitely generated $A$-module with $\operatorname{projdim}_{Q} M$ finite. 
We will not change $M$ throughout our discussion. Let $I$ be an ideal in $A$. Let $\mathscr{R}(I)=\bigoplus_{n \geq 0} I^{n} X^{n}$ be the Rees algebra of $I$. We consider $\mathscr{R}(I)$ as a subring of the polynomial ring $A[X]$. Let $N=\bigoplus N_{n}$ be a finitely generated $\mathscr{R}(I)$-module.

Set

$$
\mathscr{E}(N)=\bigoplus_{i \geq 0} \bigoplus_{n \geq 0} \operatorname{Ext}_{A}^{i}\left(M, N_{n}\right)
$$

In this section we show $\mathscr{E}(N)$ is a bigraded $\mathscr{S}=\mathscr{R}(I)\left[t_{1}, \ldots, t_{c}\right]$-module. The grading on $\mathscr{Y}$ is as follows: we set $\operatorname{deg} t_{j}=(0,2)$ for $j=1, \ldots, c$, and for $a \in I^{s}$ we set $\operatorname{deg} a X^{s}=(s, 0)$. We also discuss two preliminary results that we will need later in this paper.

2.1. Let $\mathbb{F}: \cdots F_{n} \rightarrow \cdots \rightarrow F_{1} \rightarrow F_{0} \rightarrow 0$ be a free resolution of $M$ as an $A$-module. Let $t_{1}, \ldots t_{c}: \mathbb{F}(+2) \rightarrow \mathbb{F}$ be the Eisenbud operators; see [Eisenbud 1980, Section 1]. Then:

(1) The $t_{i}$ are uniquely determined up to homotopy.

(2) Any two of them commute up to homotopy.

Let $T=A\left[t_{1}, \ldots, t_{c}\right]$ be a polynomial ring over $A$ with variables $t_{1}, \ldots, t_{c}$ of degree 2. Let $D$ be an $A$-module. The operators $t_{j}$ give well-defined maps

$$
t_{j}: \operatorname{Ext}_{A}^{i}(M, D) \rightarrow \operatorname{Ext}_{R}^{i+2}(M, D) \quad \text { for } 1 \leq j \leq c \text { and all } i,
$$

which turn $\operatorname{Ext}_{A}^{*}(M, D)=\bigoplus_{i \geq 0} \operatorname{Ext}_{A}^{i}(M, D)$ into a module over $T$. Furthermore, these structures depend only on $f$, are natural in both module arguments and commute with the connecting maps induced by short exact sequences.

2.2. Gulliksen $[1974,3.1]$ proved that if $\operatorname{projdim}_{Q} M$ is finite then $\operatorname{Ext}_{A}^{*}(M, D)$ is a finitely generated $T$-module. If $A$ is local and $D=k$, the residue field of $A$, Avramov [1989, 3.10] proved a converse; that is, if $\operatorname{Ext}_{A}^{*}(M, k)$ is a finitely generated $T$-module then $\operatorname{projdim}_{Q} M$ is finite. For a more general result, see [Avramov et al. 1997, 4.2].

2.3. Let $N=\bigoplus_{n \geq 0} N_{n}$ be a finitely generated module over $\mathscr{R}(I)$. Let $a \in I^{s}$. Consider $u=a X^{s} \in \mathscr{R}(I)_{s}$. The map

$$
N_{n} \stackrel{u}{\rightarrow} N_{n+s}
$$

yields a commutative diagram

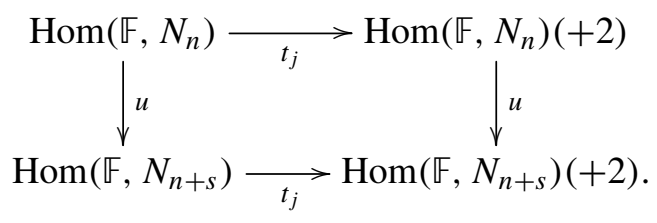


Taking homology gives that $\mathscr{E}(N)=\bigoplus \bigoplus \operatorname{Ext}_{A}^{i}\left(M, N_{n}\right)$ is a bigraded $\mathscr{S}$-module, where $\mathscr{S}=\mathscr{R}(I)\left[t_{1}, \ldots, t_{c}\right]$. $i \geq 0 n \geq 0$

Remark 2.4. (1) For each $i$, the $\mathscr{R}(I)$-module $\bigoplus \operatorname{Ext}_{A}^{i}\left(M, N_{n}\right)$ is finitely generated. $n \geq 0$

(2) For each $n$, the $A\left[t_{1}, \ldots, t_{c}\right]$-module $\bigoplus_{i \geq 0} \operatorname{Ext}_{A}^{i}\left(M, N_{n}\right)$ is finitely generated.

2.5. Notation. (1) Let $N=\bigoplus_{n \geq 0} N_{n}$ be a graded $\mathscr{R}(I)$-module. Fix $j \geq 0$. Set

$$
N_{\geq j}=\bigoplus_{n \geq j} N_{n}
$$

$\mathscr{E}\left(N_{\geq j}\right)$ is naturally isomorphic to the submodule

$$
\mathscr{E}(N)_{\geq j}=\bigoplus_{i \geq 0} \bigoplus_{n \geq j}^{\mathscr{E}(N)_{i j}}
$$

of $\mathscr{E}(N)$.

(2) If $A \rightarrow A^{\prime}$ is a ring extension and if $D$ is an $A$-module then set $D^{\prime}=D \otimes_{A} A^{\prime}$. Notice that if $D$ is a finitely generated $A$-module then $D^{\prime}$ is a finitely generated $A^{\prime}$-module.

(3) Set $\mathscr{Y}^{\prime}=\mathscr{Y} \otimes_{A} A^{\prime}$. Notice that $\mathscr{Y}^{\prime}$ is a finitely generated bigraded $A^{\prime}$-algebra. Let $U=\bigoplus_{i \geq 0} \bigoplus_{n \geq 0} U_{i, n}$ be a graded $\mathscr{S}$-module. Then

$$
U^{\prime}=U \otimes_{A} A^{\prime}=\bigoplus_{i \geq 0} \bigoplus_{n \geq 0} U_{i, n}^{\prime}
$$

is a graded $\mathscr{Y}^{\prime}$-module.

We state two lemmas that will help us in proving Theorem 1.1.

Lemma 2.6. If $\mathscr{E}\left(N_{\geq j}\right)$ is a finitely generated $\mathscr{Y}$-module then $\mathscr{E}(N)$ is a finitely generated $\mathscr{S}$-module.

Proof. Set $D=\mathscr{E}(N) / \mathscr{E}\left(N_{\geq j}\right)$. We have the following exact sequence of $\mathscr{Y}$-modules

$$
0 \rightarrow \mathscr{E}\left(N_{\geq j}\right) \rightarrow \mathscr{E}(N) \rightarrow D \rightarrow 0 .
$$

Using Gulliksen's result it follows that $D$ is a finitely generated $T=A\left[t_{1}, \ldots, t_{c}\right]$ module. Since $T$ is a subring of $\mathscr{Y}$, we get that $D$ is a finitely generated $\mathscr{S}$-module. Thus if $\mathscr{E}\left(N_{\geq j}\right)$ is a finitely generated $\mathscr{Y}$-module then $\mathscr{E}(N)$ is a finitely generated $\mathscr{Y}$-module

Lemma 2.7. (Keep the notation of 2.5(3).) Let $A \rightarrow A^{\prime}$ be a faithfully flat extension of rings and let $U=\bigoplus_{i \geq 0} \bigoplus_{n \geq 0} U_{i, n}$ be a graded $\mathscr{S}$-module. If $U^{\prime}$ is a finitely generated $\mathscr{Y}^{\prime}$-module then $U$ is a finitely generated $\mathscr{S}$-module. 
Proof. The set

$$
\mathscr{D}=\left\{u_{\text {in }} \otimes 1 \mid u_{\text {in }} \in U_{i n}, \text { where } i, n \geq 0\right\}
$$

generates $U^{\prime}$ as a $\mathscr{S}^{\prime}$-module. As $U^{\prime}$ is a finitely generated $\mathscr{S}^{\prime}$-module, we can choose a finite subset $\mathscr{C}$ of $\mathscr{D}$ which generates $U^{\prime}$ as a $\mathscr{S}^{\prime}$-module. Let

$$
V=\langle u \mid u \otimes 1 \in \mathscr{C}\rangle \text {. }
$$

Then $V$ is a finitely generated submodule of $U$. Notice that $U^{\prime}=V^{\prime}$. Thus $(U / V) \otimes_{A} A^{\prime}=0$. Since $A^{\prime}$ is a faithfully flat $A$-algebra we get $U=V$. So $U$ is a finitely generated $\mathscr{S}$-module.

\section{The local case}

In this section we prove Theorem 1.1 when $(Q, \mathfrak{n})$ is local. Let $\mathfrak{m}$ be the maximal ideal of $A$. Set $k=A / \mathfrak{m}$. Let $I$ be an ideal in $A$. Let

$$
F(I)=\mathscr{R}(I) \otimes_{A} k=\bigoplus_{n \geq 0} I^{n} / \mathfrak{m} I^{n}
$$

be the fiber cone of $I$.

3.1. Assume $N=\bigoplus_{n \geq 0} N_{n}$ is a finitely generated $\mathscr{R}(I)$-module. Notice that

$$
F(N)=N \otimes_{A} k=\bigoplus_{n \geq 0} N_{n} / \mathfrak{m} N_{n}
$$

is a finitely generated $F(I)$-module. Define

$$
\operatorname{spread}(N):=\operatorname{dim}_{F(I)} N / \mathfrak{m} N .
$$

Proof of Theorem 1.1 in the local case.

Case 1: The residue field $k=A / \mathfrak{m}$ is infinite. We induct on $\operatorname{spread}(N)$. First assume $\operatorname{spread}(N)=0$. This implies that $N_{n} / \mathfrak{m} N_{n}=0$ for all $n \gg 0$. By Nakayama's lemma, $N_{n}=0$ for all $n \gg 0$; say $N_{n}=0$ for all $n \geq j$. Then $\mathscr{E}\left(N_{\geq j}\right)=0$ and it is obviously a finitely generated $\mathscr{Y}$-module. By Lemma 2.6 we get that $\mathscr{E}(N)$ is a finitely generated $\mathscr{S}$-module.

When $\operatorname{spread}(N)>0$ then there exists $u=x t \in \mathscr{R}(I)_{1}$ which is $(N \oplus F(N))$ filter-regular, that is, there exists $j$ such that

$$
\left(0:{ }_{N} u\right)_{n}=0 \quad \text { and } \quad\left(0:{ }_{F(N)} u\right)_{n}=0 \quad \text { for all } n \geq j .
$$

Set $N_{\geq j}=\bigoplus_{n \geq j} N_{n}$ and $U=N_{\geq j} / u N_{\geq j}$. We have an exact sequence of $\mathscr{R}(I)$ modules

$$
0 \rightarrow N_{\geq j}(-1) \stackrel{u}{\rightarrow} N_{\geq j} \rightarrow U \rightarrow 0 .
$$


For each $n \geq j$ the functor $\operatorname{Hom}_{A}(M,-)$ induces the long exact sequence of $A$-modules

$$
\begin{aligned}
& 0 \rightarrow \operatorname{Hom}_{A}\left(M, N_{n}\right) \stackrel{u}{\rightarrow} \operatorname{Hom}_{A}\left(M, N_{n+1}\right) \rightarrow \operatorname{Hom}_{A}\left(M, U_{n+1}\right) \\
& \rightarrow \operatorname{Ext}_{A}^{1}\left(M, N_{n}\right) \stackrel{u}{\rightarrow} \operatorname{Ext}_{A}^{1}\left(M, N_{n+1}\right) \rightarrow \operatorname{Ext}_{A}^{1}\left(M, U_{n+1}\right) \\
& \rightarrow \quad \ldots \quad \stackrel{u}{\rightarrow} \quad \ldots \quad \rightarrow \quad \ldots \\
& \rightarrow \operatorname{Ext}_{A}^{i}\left(M, N_{n}\right) \stackrel{u}{\rightarrow} \operatorname{Ext}_{A}^{i}\left(M, N_{n+1}\right) \rightarrow \operatorname{Ext}_{A}^{i}\left(M, U_{n+1}\right) \\
& \rightarrow \quad \ldots \quad \stackrel{u}{\rightarrow} \quad \ldots \quad \rightarrow \quad \ldots
\end{aligned}
$$

Using the naturality of Eisenbud operators we have the following exact sequence of $\mathscr{Y}$-modules

$$
\mathscr{E}\left(N_{\geq} j\right)(-1,0) \stackrel{(u, 0)}{\longrightarrow} \mathscr{E}\left(N_{\geq} j\right) \rightarrow \mathscr{E}(U) .
$$

By construction,

$$
\operatorname{spread}(U)=\operatorname{spread}\left(N_{\geq j}\right)-1=\operatorname{spread}(N)-1 .
$$

By the induction hypothesis, $\mathscr{E}(U)$ is a finitely generated $\mathscr{Y}$-module. Therefore by Lemma 3.2 we get $\mathscr{E}\left(N_{\geq j}\right)$ is a finitely generated $\mathscr{S}$-module. Using Lemma 2.6 we get that $\mathscr{E}(N)$ is a finitely generated $\mathscr{Y}$-module.

Case 2: The residue field $k$ is finite.

In this case we do the standard trick. Let $Q^{\prime}=Q[X]_{\mathfrak{n} Q[X]}$. Set $A^{\prime}=A \otimes_{Q} Q^{\prime}$. Notice that $A^{\prime}=A[X]_{\mathfrak{m} A[X]}$ is a flat $A$-algebra with residue field $k(X)$ which is infinite. Notice that $f_{1}, \ldots, f_{c}$ is a $Q^{\prime}$-regular sequence and $Q^{\prime} /(\boldsymbol{f})=A^{\prime}$. Set $I^{\prime}=I A^{\prime}$ and $M^{\prime}=M \otimes_{Q} Q^{\prime}=M \otimes_{A} A^{\prime}$. Notice that $\operatorname{projdim}_{Q^{\prime}} M^{\prime}$ is finite. Set $\mathscr{R}(I)^{\prime}=\mathscr{R}\left(I^{\prime}\right)$, the Rees algebra of $I^{\prime}$. Then $N^{\prime}=N \otimes_{A} A^{\prime}$ is a finitely generated $\mathscr{R}(I)^{\prime}$-module. Also note that $\mathscr{E}\left(N^{\prime}\right)=\mathscr{E}(N) \otimes_{A} A^{\prime}$.

By Case 1 we have that $\mathscr{E}\left(N^{\prime}\right)$ is a finitely generated $\mathscr{S}^{\prime}$-module. So by Lemma 2.7 we get that $\mathscr{E}(N)$ is a finitely generated $\mathscr{Y}$-module.

The next lemma is a bigraded version of Lemma 2.8(1) of [Puthenpurakal 2005].

Lemma 3.2. Let $R$ be a Noetherian ring (not necessarily local) and let $B=$ $\bigoplus_{i, j \geq 0} B_{i, j}$ be a finitely generated bigraded $R$-algebra with $B_{0,0}=R$. Note that $B$ need not be standard graded. Set

$$
B_{y}=\bigoplus_{j \geq 0} B_{(0, j)} .
$$

Let $V=\bigoplus_{i, j \geq 0} V_{i, j}$ be a bigraded $B$-module satisfying these conditions:

(1) For each $i \geq 0, V_{i}=\bigoplus_{j \geq 0} V_{i, j}$ is finitely generated as a $B_{y}$-module. 
(2) There exists $z \in B_{(r, 0)}$ (with $r \geq 1$ ) and a finitely generated bigraded $B$-module $D$ such that we have an exact sequence of $B$-modules

$$
V(-r, 0) \stackrel{z}{\rightarrow} V \stackrel{\psi}{\rightarrow} D
$$

Then $V$ is a finitely generated B-module.

Proof. Step 1. We begin by reducing to the case when $\psi$ is surjective. Notice that $D^{\prime}=$ image $\psi$ is a finitely generated bigraded $B$-module. If $\psi^{\prime}: V \rightarrow D^{\prime}$ is the map induced by $\psi$ then we have an exact sequence

$$
V(-r, 0) \stackrel{z}{\rightarrow} V \stackrel{\psi^{\prime}}{\rightarrow} D^{\prime} \rightarrow 0 .
$$

Thus we may assume $\psi$ is surjective.

Step 2. Choosing generators:

2.1. Choose a finite set $W$ in $V$ of homogeneous elements such that

$$
\psi(W)=\{\psi(w) \mid w \in W\}
$$

is a generating set for $D$.

2.2. Assume all the elements in $W$ have $x$-coordinate $\leq c$.

2.3. For each $i \geq 0$, by hypothesis, $V_{i}$ is a finitely generated $B_{y}$-module. So we may choose a finite set $P_{i}$ of homogeneous elements in $V_{i}$ which generates $V_{i}$ as a $B_{y}$-module.

2.4. Set

$$
G=W \cup\left(\bigcup_{i=0}^{c} P_{i}\right) .
$$

Clearly $G$ is a finite set.

Claim. $G$ is a generating set for $V$.

Let $U$ be the $B$-submodule of $V$ generated by $G$. It suffices to prove that $U_{i, j}=V_{i, j}$ for all $i, j \geq 0$. By construction we have that for $0 \leq i \leq c$

$$
U_{i, j}=V_{i, j} \quad \text { for each } j \geq 0 .
$$

We give $X:=\mathbb{Z}_{\geq 0} \times \mathbb{Z}_{\geq 0}$ the lex-order $\preceq$, making it well ordered. So we can prove our result by induction on $X$ with respect to $\preceq$.

The base case is $(0,0)$. In this case $U_{0,0}=V_{0,0}$ by (*). Let $(i, j) \in X \backslash\{(0,0)\}$ and assume that for all $(r, s) \prec(i, j)$ we have $U_{r, s}=V_{r, s}$.

Subcase 1: $i \leq c$. By $\left(^{*}\right)$ we have $U_{i, j}=V_{i, j}$. 
Subcase 2: $i>c$. Let $p \in V_{i, j}$. By construction, there exist $w_{1}, \ldots, w_{m} \in W \subseteq G$ such that

$$
\psi(p)=\sum_{l=1}^{m} h_{l} \psi\left(w_{l}\right), \quad \text { where } h_{l} \in B .
$$

We may assume that $\operatorname{deg} h_{l} w_{l}=(i, j)$ for each $l$. Set $p^{\prime}=\sum_{l=1}^{m} h_{l} w_{l} \in V_{i, j}$. Then $p^{\prime} \in U_{i, j}$ and $p-p^{\prime} \in \operatorname{ker} \psi$. So

$$
p-p^{\prime}=z \cdot q, \quad \text { where } q \in V_{(i-r, j)} .
$$

If $q=0$ then $p=p^{\prime} \in U_{i, j}$. Otherwise, note that $(i-r, j) \prec(i, j)$. So by induction hypothesis, $q \in U_{(i-r, j)}$. It follows that $p \in U_{i, j}$. Thus $V_{i, j} \subseteq U_{i, j}$. Since $U_{i, j} \subseteq V_{i, j}$, by construction it follows that $U_{i, j}=V_{i, j}$. The result follows by induction on $X$.

\section{The global case}

We need quite a few preliminaries to prove the global case of Theorem 1.1. See Section 4.2 for the difficulty in going from the local to the global case. Note that in the local case we proved the result by inducting on $\operatorname{spread}(N)$. This is unavailable to us in the global situation as there are usually infinitely many maximal ideals in a global ring. Most of this section will discuss two invariants of a graded $\mathscr{R}(I)$-module $N=\bigoplus_{n \geq 0} N_{n}$. We will use these invariants to prove Theorem 1.1 by induction.

4.1. Notation and conventions. We take the dimension of the zero-module to be -1 . We also set the degree of the zero-polynomial to be -1 .

Let $\mathfrak{P} \in \operatorname{Spec} Q$. If $\mathfrak{P} \supseteq \boldsymbol{f}$ then set $\mathfrak{p}=\mathfrak{P} / \boldsymbol{f}$. If $\mathfrak{P} \nsupseteq \boldsymbol{f}$ then any $A$-module localized at $\mathfrak{P}$ is zero. So assume $\mathfrak{P} \supseteq \boldsymbol{f}$.

(1) $\mathscr{R}(I)_{\mathfrak{p}} \cong \mathscr{R}\left(I A_{\mathfrak{p}}\right)$ and $\mathscr{S}_{\mathfrak{p}} \cong \mathscr{R}(I)_{\mathfrak{p}}\left[t_{1}, \ldots, t_{c}\right]$.

(2) $M_{\mathfrak{p}}=M_{\mathfrak{P}}$ has finite projective dimension as a $Q_{\mathfrak{P}}$-module.

(3) $\mathscr{E}(N)_{\mathfrak{p}} \cong \mathscr{E}\left(N_{\mathfrak{p}}\right)$.

4.2. The difficulty in going from local to global. For each $\mathfrak{p} \in \operatorname{Spec} A$ it follows from Section 4.1 that $\mathscr{E}\left(N_{\mathfrak{p}}\right)$ is a finitely generated $\mathscr{Y}_{\mathfrak{p}}$-module. Usually $\operatorname{Supp}_{A} \mathscr{E}(N)$ will be an infinite set. So we cannot apply the local case and conclude.

The situation when $\operatorname{Supp}_{A} \mathscr{E}(N)$ is a finite set will help in the base step of our induction argument to prove Theorem 1.1. So we show it separately.

Lemma 4.3. If $\operatorname{Supp}_{A} \mathscr{E}(N)$ is a finite set then $\mathscr{E}(N)$ is a finitely generated $\mathscr{S}$ module.

Proof. We may choose a finite subset $C$ of $\mathscr{E}(N)$ such that its image in $\mathscr{E}(N)_{\mathfrak{p}}$ generates $\mathscr{E}(N)_{\mathfrak{p}}$ for each $\mathfrak{p} \in \operatorname{Supp}_{A} \mathscr{E}(N)$. Set $U$ to be the finitely generated submodule of $\mathscr{E}(N)$ generated by $C$. 
Set $D=\mathscr{E}(N) / U$. Notice that $D_{\mathfrak{p}}=0$ for each $\mathfrak{p} \in \operatorname{Spec} A$. So $D=0$. Therefore $\mathscr{E}(N)=U$ is a finitely generated $\mathscr{S}$-module.

4.4. First inductive device. Since $N$ is a finitely generated $\mathscr{R}(I)$-module we have $\operatorname{ann}_{A} N_{n} \subseteq \operatorname{ann}_{A} N_{n+1}$ for all $n \gg 0$. Since $A$ is Noetherian it follows that $\operatorname{ann}_{A} N_{n}$ is constant for all $n \gg 0$. Call this stable value $\mathfrak{L}_{N}$. This enables us to define the limit dimension of $N$.

$$
\lim \operatorname{dim} N=\lim _{n \rightarrow \infty} \operatorname{dim}_{A} N_{n}=\operatorname{dim} A / \mathfrak{L}_{N} .
$$

Since $A$ has finite Krull dimension we get that $\lim \operatorname{dim} N$ is finite.

4.5. Let $\mathfrak{P}$ be a prime ideal in $A$. If $D$ is a finitely generated $A$-module then

$$
\operatorname{ann}_{A_{\mathfrak{P}}} D_{\mathfrak{P}}=\left(\operatorname{ann}_{A} D\right)_{\mathfrak{P}}=\left(\operatorname{ann}_{A} D\right) A_{\mathfrak{P}}
$$

Therefore

$$
\left(\mathfrak{L}_{N}\right)_{\mathfrak{P}}=\mathfrak{L}_{N_{\mathfrak{P}}} .
$$

4.6. Note that if $\lim \operatorname{dim}(N)=-1$ then $N_{j}=0$, say for all $j \geq j_{0}$. So $\mathscr{E}\left(N_{\geq j_{0}}\right)=0$. Using Lemma 2.6 it follows that $\mathscr{E}(N)$ is a finitely generated $\mathscr{Y}$-module. The first nontrivial case is the following:

Proposition 4.7. If $\lim \operatorname{dim}(N)=0$ then $\mathscr{E}(N)$ is a finitely generated $\mathscr{S}$-module.

Proof. This implies that $A / \mathfrak{L}_{N}$ is Artinian. Say $\operatorname{dim} N_{n}=0$ for $n \geq r$. Clearly,

$$
\operatorname{Supp}_{A} \mathscr{E}\left(N_{\geq r}\right) \subseteq \operatorname{Supp}_{A} A / \mathfrak{L}_{N},
$$

a finite set of maximal ideals in $A$. It follows from Lemma 4.3 that $\mathscr{E}\left(N_{\geq r}\right)$ is a finitely generated $\mathscr{S}$-module. Using Lemma 2.6 we get that $\mathscr{E}(N)$ is a finitely generated $\mathscr{S}$-module.

4.8. Higher-degree filter-regular element. We do not have filter-regular elements of degree 1 in the global situation. However we can do the following:

Set $E=N / H_{R_{+}}^{0}(N)$. Assume $E \neq 0$. As $H_{R_{+}}^{0}(E)=0$ there exists homogeneous $u \in R_{+}$such that $u$ is $E$-regular [Bruns and Herzog 1993, 1.5.11]. Say deg $u=s$. Since $E_{n}=N_{n}$ for all $n \gg 0$ it follows that the map $N_{i} \rightarrow N_{i+s}$ induced by multiplication by $u$ is injective for all $i \gg 0$. We will say that $u$ is an $N$ filter-regular element of degree $s$.

4.9. The second inductive device. We now discuss a global invariant of $N$ which patches well with local ones. 
4.10. The local invariant. Let $(A, \mathfrak{m})$ be local and let $W=\bigoplus_{n \geq 0} W_{n}$ be a finitely generated $\mathscr{R}(I)$-module. Suppose $\mathfrak{L}_{W}=\operatorname{ann}_{A} W_{n}$ for all $n \geq c$. Let $\mathfrak{a} \subseteq \mathfrak{L}_{W}$ be an ideal. Fix $j \geq 0$. Set

$$
d_{\mathfrak{a}}(W, j)= \begin{cases}0 & \text { if } j<c, \\ 0 & \text { if } j \geq c \text { and } \operatorname{dim} W_{j}<\operatorname{dim} A / \mathfrak{a}, \\ e\left(\mathfrak{m}, W_{j}\right) & \text { otherwise. }\end{cases}
$$

Note that for $j \geq c, W_{j}$ is an $A / \mathfrak{a}$-module. Furthermore $d_{\mathfrak{a}}(W, j)$ is the modified multiplicity function on the $A / \mathfrak{a}$-module $W_{j}$.

Remark 4.11. Notice if $\operatorname{dim} W_{j}=\operatorname{dim} A / \mathfrak{a}$ and $j \geq c$ then

$$
d_{\mathfrak{a}}(W, j)=d_{\mathfrak{L}_{W}}(W, j) .
$$

Let $\mu(D)$ denote the minimal number of generators of an $A$-module $D$.

Lemma 4.12. The function $d_{\mathfrak{a}}(W,-)$ is of polynomial type of degree $\leq \mu(I)-1$.

Proof. We may assume that the residue field of $A$ is infinite. Set $T=\mathscr{R}(I) / \mathfrak{a} \mathscr{R}(I)=$ $\bigoplus_{n \geq 0} T_{n}$. Notice $T_{0}=A / \mathfrak{a}$. Let $\boldsymbol{x}=x_{1}, \ldots, x_{r}$ be a minimal reduction of $\mathfrak{m}(A / \mathfrak{a})$. So $e(\mathfrak{m},-)=e(\boldsymbol{x},-)$ [Bruns and Herzog 1993, 4.6.5]. By a result due to Serre [Bruns and Herzog 1993, 4.7.6], we get that

$$
e\left(\boldsymbol{x}, W_{j}\right)=\sum_{i=0}^{r}(-1)^{i} \ell\left(H_{i}\left(\boldsymbol{x}, W_{j}\right)\right) .
$$

Notice $H_{i}(\boldsymbol{x}, W)=\bigoplus_{j \geq c} H_{i}\left(\boldsymbol{x}, W_{j}\right)$ is a finitely generated $T / \boldsymbol{x} T$-module. Notice $(T / x T)_{0}=A /(\mathfrak{a}+\boldsymbol{x})$ is Artinian. Furthermore $(T / \boldsymbol{x} T)_{1}$ is a quotient of $\mathscr{R}(I)_{1}$ and so can be generated by $\mu(I)$ elements. Therefore the function $j \mapsto \ell\left(H_{i}\left(\boldsymbol{x}, W_{j}\right)\right)$ is of polynomial type of degree $\leq \mu(I)-1$. The result follows.

Definition 4.13. $\theta(\mathfrak{a}, W)$ is the degree of the polynomial function $d_{\mathfrak{a}}(W,-)$.

Remark 4.14. Clearly $\theta(\mathfrak{a}, W)$ is nonnegative if and only if $\lim \operatorname{dim} W=\operatorname{dim} R / \mathfrak{a}$ and is -1 otherwise. Note that if $\operatorname{dim} A / \mathfrak{a}=\lim \operatorname{dim} W$ then $\theta(\mathfrak{a}, W)=\theta\left(\mathfrak{L}_{W}, W\right)$ is independent of $\mathfrak{a}$.

4.15. The global invariant. Let $A$ be a Noetherian ring with finite Krull dimension. Let $I=\left(x_{1}, \ldots, x_{s}\right)$ be an ideal in $A$. Let $W=\bigoplus_{n \geq 0} W_{n}$ be a finitely generated $\mathscr{R}(I)$-module. We assume that $\mathfrak{L}_{W}=\operatorname{ann}_{A} W_{n}$ for all $n \geq c$. Let $\mathfrak{a} \subseteq \mathfrak{L}_{W}$ be an ideal.

Set

$$
\mathscr{C}(\mathfrak{a})=\left\{\mathfrak{m} \mid \mathfrak{m} \in \mathfrak{m}-\operatorname{Spec}(A), \mathfrak{m} \supseteq \mathfrak{a} \text { and } \operatorname{dim}(A / \mathfrak{a})_{\mathfrak{m}}=\operatorname{dim} A / \mathfrak{a}\right\} .
$$

Let $I=\left(x_{1}, \ldots, x_{s}\right)$. If $\mathfrak{m} \in \mathscr{C}(\mathfrak{a})$ we have:

(a) $W_{\mathfrak{m}}=\bigoplus_{n \geq 0}\left(W_{n}\right)_{\mathfrak{m}}$. 
(b) $\mathfrak{L}_{W_{\mathfrak{m}}}=\left(\mathfrak{L}_{W}\right)_{\mathfrak{m}}$. So $\mathfrak{a}_{\mathfrak{m}} \subseteq \mathfrak{L}_{W_{\mathfrak{m}}}$.

(c) $\theta\left(\mathfrak{a}_{\mathfrak{m}}, W_{\mathfrak{m}}\right) \leq s-1$.

Define

$$
\theta(\mathfrak{a}, W)=\max \left\{\theta\left(\mathfrak{a}_{\mathfrak{m}}, W_{\mathfrak{m}}\right) \mid \mathfrak{m} \in \mathscr{b}(\mathfrak{a})\right\} .
$$

By (c) above we get that $\theta(\mathfrak{a}, W)$ is finite and is $\leq s-1$.

4.16. Properties of $\boldsymbol{\theta}(\mathfrak{a}, \boldsymbol{W})$. We describe some properties of $\theta(\mathfrak{a}, W)$ we need for the proof of the global case of Theorem 1.1. Let $I=\left(x_{1}, \ldots, x_{s}\right)$.

(i) $\theta(\mathfrak{a}, W) \leq s-1$. This is clear.

(ii) If $\mathfrak{L}_{W} \neq A$ then $\theta\left(\mathfrak{L}_{W}, W\right) \geq 0$. It suffices to consider the local case. Note that then $d_{\mathfrak{L}_{W}}(W, j)>0$ for all $j \geq c$. It follows that $\theta\left(\mathfrak{L}_{W}, W\right) \geq 0$.

(iii) $\theta(\mathfrak{a}, W)=-1$ if and only if $\lim \operatorname{dim} W<\operatorname{dim} A / \mathfrak{a}$. If $\theta(\mathfrak{a}, W)=-1$ then $\theta\left(\mathfrak{a}_{\mathfrak{m}}, W_{\mathfrak{m}}\right)=-1$ for all $\mathfrak{m} \in \mathscr{C}(\mathfrak{a})$. This is equivalent to saying that $\lim \operatorname{dim} W_{\mathfrak{m}}<$ $\operatorname{dim}(A / \mathfrak{a})_{\mathfrak{m}}$ for all $\mathfrak{m} \in \mathscr{C}(\mathfrak{a})$. By definition of $\mathscr{C}(\mathfrak{a})$ we have that

$$
\operatorname{dim} A / \mathfrak{a}=\operatorname{dim}(A / \mathfrak{a})_{\mathfrak{m}} \quad \text { for each } \mathfrak{m} \in \mathscr{C}(\mathfrak{a}) .
$$

Also note that as $\mathfrak{a} \subseteq \mathfrak{L}_{W}$ we have

$$
\lim \operatorname{dim} W=\max \left\{\lim \operatorname{dim} W_{\mathfrak{m}} \mid \mathfrak{m} \in \mathscr{C}(\mathfrak{a})\right\} .
$$

So $\lim \operatorname{dim} W<\operatorname{dim} A / \mathfrak{a}$.

Conversely if $\lim \operatorname{dim} W<\operatorname{dim} A / \mathfrak{a}$ then for all $\mathfrak{m} \in \mathscr{C}(\mathfrak{a})$ we have

$$
\lim \operatorname{dim} W_{\mathfrak{m}} \leq \lim \operatorname{dim} W<\operatorname{dim} A / \mathfrak{a}=\operatorname{dim}(A / \mathfrak{a})_{\mathfrak{m}} .
$$

So $\theta\left(\mathfrak{a}_{\mathfrak{m}}, W_{\mathfrak{m}}\right)=-1$ for all $\mathfrak{m} \in \mathscr{C}(\mathfrak{a})$. Thus $\theta(\mathfrak{a}, W)=-1$.

(iv) If $\theta(\mathfrak{a}, W) \geq 0$ then $\theta\left(\mathfrak{L}_{W}, W\right) \leq \theta(\mathfrak{a}, W)$. By (iii) we get that $\lim \operatorname{dim} W=$ $\operatorname{dim} A / \mathfrak{a}$. By hypothesis we also have $\mathfrak{a} \subseteq \mathfrak{L}_{W}$. Since $\operatorname{dim} A / \mathfrak{a}=\operatorname{dim} A / \mathfrak{L}_{W}$ it follows that $\mathscr{C}\left(\mathfrak{L}_{W}\right) \subseteq \mathscr{C}(\mathfrak{a})$. Using Remark 4.11 it follows that $\theta\left(\mathfrak{L}_{W}, W\right) \leq \theta(\mathfrak{a}, W)$.

(v) Let $u \in \mathscr{R}(I)_{+}$be homogeneous of degree $b$. Assume $u$ is $W$-filter regular and $W_{n} \neq 0$ for all $n \gg 0$. Set $E=W / u W$. Notice that $\mathfrak{L}_{W} \subseteq \mathfrak{L}_{E}$. Then

$$
\theta\left(\mathfrak{L}_{W}, E\right) \leq \theta\left(\mathfrak{L}_{W}, W\right)-1 .
$$

We have nothing to show if $\theta\left(\mathfrak{L}_{W}, E\right)=-1$. So assume $\theta\left(\mathfrak{L}_{W}, E\right) \geq 0$. Suppose $\theta\left(\mathfrak{L}_{W}, E\right)=\theta\left(\left(\mathfrak{L}_{W}\right)_{\mathfrak{p}}, E_{\mathfrak{p}}\right)$ for some $\mathfrak{p} \in \mathscr{C}\left(\mathfrak{L}_{W}\right)$. Since $u$ is $W$-filter-regular, multiplication by $u$ induces the exact sequence

$$
0 \rightarrow W_{j-b} \rightarrow W_{j} \rightarrow E_{j} \rightarrow 0 \text { for all } j \gg 0 .
$$


Localization at $\mathfrak{p}$ yields an exact sequence

$$
0 \rightarrow\left(W_{j-b}\right)_{\mathfrak{p}} \rightarrow\left(W_{j}\right)_{\mathfrak{p}} \rightarrow\left(E_{j}\right)_{\mathfrak{p}} \rightarrow 0 \text { for all } j \gg 0 .
$$

Since $d_{\mathfrak{L}_{W \mathfrak{p}}}(-,-)$ is an additive functor on $\left(A / \mathfrak{L}_{W}\right)_{\mathfrak{p}}$-modules we get that

$$
\theta\left(\left(\mathfrak{L}_{W}\right)_{\mathfrak{p}}, E_{\mathfrak{p}}\right)=\theta\left(\left(\mathfrak{L}_{W}\right)_{\mathfrak{p}}, W_{\mathfrak{p}}\right)-1 .
$$

The result follows since

$$
\theta\left(\left(\mathfrak{L}_{W}\right)_{\mathfrak{p}}, E_{\mathfrak{p}}\right)=\theta\left(\mathfrak{L}_{W}, E\right) \quad \text { and } \quad \theta\left(\left(\mathfrak{L}_{W}\right)_{\mathfrak{p}}, W_{\mathfrak{p}}\right) \leq \theta\left(\mathfrak{L}_{W}, W\right) .
$$

Proof of Theorem 1.1. We induct on $\lim \operatorname{dim} N$. If $\lim \operatorname{dim} N=-1,0$ then the result follows from Section 4.6 and Proposition 4.7.

Assume $\lim \operatorname{dim} N \geq 1$ and assume the result holds for all $\mathscr{R}(I)$-modules $E$ with $\lim \operatorname{dim} E \leq \lim \operatorname{dim} N-1$. Let $x \in \mathscr{R}(I)_{+}$be homogeneous and an $N$-filter-regular element. Let $\operatorname{deg} x=r$. Set $D=N / x D$. By Lemma 2.6 it suffices to assume the case when $x$ is $N$-regular.

We now induct on $\theta\left(\mathfrak{L}_{N}, N\right)$. If $\theta\left(\mathfrak{L}_{N}, N\right)=0$ then $\theta\left(\mathfrak{L}_{N}, D\right) \leq-1$, by Section 4.16(v). Using Section 4.16(iii) we get that

$$
\lim \operatorname{dim} D<\operatorname{dim} A / \mathfrak{L}_{N}=\lim \operatorname{dim} N .
$$

By the induction hypothesis (on lim $\operatorname{dim}$ ) the module $\mathscr{E}(D)$ is a finitely generated $\mathscr{S}$-module. The short exact sequence of $\mathscr{R}(I)$-modules

$$
0 \rightarrow N(-r) \stackrel{x}{\rightarrow} N \rightarrow D \rightarrow 0
$$

induces an exact sequence of $\mathscr{Y}$-modules

$$
\mathscr{E}(N)(-r, 0) \stackrel{x}{\rightarrow} \mathscr{E}(N) \rightarrow \mathscr{E}(D) .
$$

By Lemma 3.2 we get that $\mathscr{E}(N)$ is a finitely generated $\mathscr{S}$-module.

We assume the result if $\theta\left(\mathfrak{L}_{N}, N\right) \leq i$ and prove it when $\theta\left(\mathfrak{L}_{N}, N\right)=i+1$. Let $D$ be as above. So $\theta\left(\mathfrak{L}_{N}, D\right) \leq i$, by Section 4.16(v). If $\theta\left(\mathfrak{L}_{N}, D\right)=-1$ then the argument as above yields $\mathscr{E}(N)$ to be a finitely generated $\mathscr{S}$-module.

If $\theta\left(\mathfrak{L}_{N}, D\right) \geq 0$ then by Section 4.16(iv) we get that $\theta\left(\mathfrak{L}_{D}, D\right) \leq \theta\left(\mathfrak{L}_{N}, D\right) \leq i$. So by induction hypothesis on $\theta(-,-)$ we get that $\mathscr{E}(D)$ is a finitely generated $\mathscr{S}$-module. By an argument similar to the one above we get that $\mathscr{E}(N)$ is a finitely generated $\mathscr{S}$-module.

\section{Application I: Asymptotic associated primes - the local case}

In this section we give an answer to our main motivating question.

Theorem 5.1. Let $(A, \mathfrak{m})$ be a local complete intersection. Let $M$ be a finitely generated A-module. Let I be an ideal in $A$ and let $N=\bigoplus_{n \geq 0} N_{n}$ be a finitely 
generated $\mathscr{R}(I)$-module. Then

$$
\bigcup_{n \geq 0} \bigcup_{i \geq 0} \operatorname{Ass}_{A} \operatorname{Ext}_{A}^{i}\left(M, N_{n}\right) \text { is a finite set. }
$$

Furthermore there exist $i_{0}, n_{0}$ such that for all $i \geq i_{0}$ and $n \geq n_{0}$ we have

$$
\begin{aligned}
\operatorname{Ass}_{A} \operatorname{Ext}_{A}^{2 i}\left(M, N_{n}\right) & =\operatorname{Ass}_{A} \operatorname{Ext}_{A}^{2 i_{0}}\left(M, N_{n_{0}}\right), \\
\operatorname{Ass}_{A} \operatorname{Ext}_{A}^{2 i+1}\left(M, N_{n}\right) & =\operatorname{Ass}_{A} \operatorname{Ext}_{A}^{2 i_{0}+1}\left(M, N_{n_{0}}\right) .
\end{aligned}
$$

Recall a local ring $A$ is said to be a complete intersection if $\hat{A}=Q /\left(f_{1}, \ldots, f_{c}\right)$, where $(Q, \mathfrak{n})$ is a complete regular local ring and $f$ is a $Q$-regular sequence. If $A$ is a complete intersection and a quotient of a regular local ring $T$ then it can be shown that $A=T /\left(g_{1}, \ldots, g_{c}\right)$, where $\boldsymbol{g}$ is a $T$-regular sequence (see [Matsumura 1980, 21.2]). In this case Theorem 5.1 holds by the following more general result:

Theorem 5.2. Let $Q$ be a Noetherian ring with finite Krull dimension and let $\boldsymbol{f}=$ $f_{1}, \ldots f_{c}$ be a regular sequence in $Q$. Set $A=Q /(\boldsymbol{f})$. Let $M$ be a finitely generated $A$-module with projdim $_{Q} M$ finite. Let I be an ideal in A and let $N=\bigoplus_{n \geq 0} N_{n}$ be a
finitely generated $\mathscr{R}(I)$-module. Then

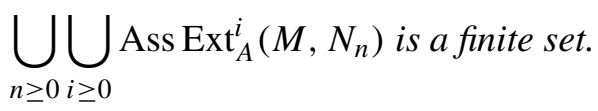

Furthermore there exist $i_{0}, n_{0}$ such that for all $i \geq i_{0}$ and $n \geq n_{0}$ we have

$$
\begin{aligned}
\operatorname{Ass}_{A} \operatorname{Ext}_{A}^{2 i}\left(M, N_{n}\right) & =\operatorname{Ass}_{A} \operatorname{Ext}_{A}^{2 i_{0}}\left(M, N_{n_{0}}\right), \\
\operatorname{Ass}_{A} \operatorname{Ext}_{A}^{2 i+1}\left(M, N_{n}\right) & =\operatorname{Ass}_{A} \operatorname{Ext}_{A}^{2 i_{0}+1}\left(M, N_{n_{0}}\right) .
\end{aligned}
$$

The following example shows that two sets of stable values of associate primes can occur.

Example 5.3. Let $Q=k \llbracket u, x \rrbracket, A=Q /(u x)$. Let $M=Q /(u), I=A$ and $N=M[t]$ (so $N_{n}=M$ for all $n$ ).

For $i \geq 1$ one has (see [Avramov and Buchweitz 2000, 4.3])

$$
\operatorname{Ext}_{A}^{2 i-1}(M, M)=0 \quad \text { and } \quad \operatorname{Ext}_{A}^{2 i}(M, M)=k .
$$

5.4. We now state a special case of a result due to E. West [2004, 3.2 and 5.1].

Let $R=A\left[x_{1}, \ldots, x_{r} ; y_{1}, \ldots y_{s}\right]$ be a bigraded $A$-algebra with $\operatorname{deg} x_{i}=(2,0)$ and $\operatorname{deg} y_{j}=(0,1)$. Let $M=\bigoplus_{i, n \geq 0} M_{(i, n)}$ be a finitely generated $R$-module. Then:

(1) $\bigcup_{i \geq 0} \bigcup_{n \geq 0} \operatorname{Ass}_{A} M_{(i, n)}$ is a finite set.

(2) There exist $i_{0}, n_{0}$ such that for all $i \geq i_{0}$ and $n \geq n_{0}$ we have

$$
\operatorname{Ass}_{A} M_{(2 i, n)}=\operatorname{Ass}_{A} M_{\left(2 i_{0}, n_{0}\right)}, \quad \operatorname{Ass}_{A} M_{(2 i+1, n)}=\operatorname{Ass}_{A} M_{\left(2 i_{0}+1, n_{0}\right)} .
$$


Proof of Theorem 5.2. The result follows from our main theorem (1.1) and 5.4.

We need the following exercise problem from [Matsumura 1980, 6.7, page 42]. Fact 5.5. Let $f: A \rightarrow B$ be a ring homomorphism of Noetherian rings. Let $U$ be a finitely generated $B$-module. Then

$$
\operatorname{Ass}_{A} U=\left\{\mathfrak{P} \cap A \mid \mathfrak{P} \in \operatorname{Ass}_{B} U\right\} .
$$

In particular $\mathrm{Ass}_{A} U$ is a finite set.

There exist complete intersection rings which are not quotients of a regular local ring (see [Heitmann and Jorgensen 2012]). So Theorem 5.2 does not settle Theorem 5.1. To prove an analog of Theorem 5.2 for a local complete intersection we need the following result.

Lemma 5.6. Let $(A, \mathfrak{m})$ be a Noetherian local ring. Let $\hat{A}$ be the completion of $A$ with respect to $\mathfrak{m}$. Let $B$ be a finitely generated $\hat{A}$-algebra containing $\hat{A}$. Let $E$ be an A-module such that $E \otimes_{A} \hat{A}$ is a finitely generated $B$-module. Let $D$ be any A-module. Then:

(a) $\operatorname{Ass}_{\hat{A}} E \otimes_{A} \hat{A}$ is a finite set.

(b) $\operatorname{Ass}_{A} D=\left\{\mathfrak{P} \cap A \mid \mathfrak{P} \in \operatorname{Ass}_{\hat{A}}\left(D \otimes_{A} \hat{A}\right)\right\}$.

(c) $\operatorname{Ass}_{A} E$ is a finite set.

To prove this result we need Theorem 23.3 from [Matsumura 1980]. Unfortunately, there is a typographical error there, so we state it here.

Theorem 5.7. Let $\varphi: A \rightarrow B$ be a homomorphism of Noetherian rings, and let $E$ be an $A$-module and $G$ a $B$-module. Suppose that $G$ is flat over $A$; then we have the following:

(i) If $\mathfrak{p} \in \operatorname{Spec} A$ and $G / \mathfrak{p} G \neq 0$ then

$$
{ }^{a} \varphi\left(\operatorname{Ass}_{B}(G / \mathfrak{p} G)\right)=\operatorname{Ass}_{A}(G / \mathfrak{p} G)=\{\mathfrak{p}\} .
$$

(ii) $\operatorname{Ass}_{B}\left(E \otimes_{A} G\right)=\bigcup_{\mathfrak{p} \in \operatorname{Ass}_{A}(E)} \operatorname{Ass}_{B}(G / \mathfrak{p} G)$.

Remark 5.8. In [Matsumura 1980], $\operatorname{Ass}_{A}(E \otimes G)$ is written instead of $\operatorname{Ass}_{B}(E \otimes G)$. Also note that ${ }^{a} \varphi(\mathfrak{P})=\mathfrak{P} \cap A$ for $\mathfrak{P} \in \operatorname{Spec} B$.

Proof of Lemma 5.6. We consider the natural ring homomorphisms

$$
\alpha: A \hookrightarrow \hat{A}, \quad \beta: \hat{A} \hookrightarrow B .
$$

(a) We use the map $\beta$ and Fact 5.5 to get our result.

(b) Set $X=\left\{\mathfrak{P} \cap A \mid \mathfrak{P} \in \operatorname{Ass}_{\hat{A}}\left(D \otimes_{A} \hat{A}\right)\right\}$. We consider the flat map $\alpha$. 
Let $\mathfrak{q} \in X$. Say $\mathfrak{q}=\mathfrak{P} \cap A$, where $\mathfrak{P} \in \operatorname{Ass}_{\hat{A}} D \otimes \hat{A}$. By Theorem 5.7(ii), $\mathfrak{P} \in \operatorname{Ass}_{\hat{A}} \hat{A} / \mathfrak{p} \hat{A}$ for some $\mathfrak{p} \in \operatorname{Ass}_{A} D$. Notice $\hat{A} / \mathfrak{p} \hat{A} \neq 0$. By Theorem 5.7.(i) it follows that $\mathfrak{p}=\mathfrak{P} \cap A=\mathfrak{q}$. So $X \subseteq \operatorname{Ass}_{A} D$.

Conversely, if $\mathfrak{p} \in \operatorname{Ass}_{A} D$, then by Theorem 5.7(ii), Ass ${ }_{\hat{A}} \hat{A} / \mathfrak{p} \hat{A} \subseteq \operatorname{Ass}_{\hat{A}} D \otimes \hat{A}$. Notice $\hat{A} / \mathfrak{p} \hat{A} \neq 0$. Let $\mathfrak{P} \in \operatorname{Ass}_{\hat{A}} \hat{A} / \mathfrak{p} \hat{A}$. Then by Theorem 5.7(i) we have $\mathfrak{p}=$ $\mathfrak{P} \cap A \in X$. Thus $\operatorname{Ass}_{A} D \subseteq X$. It follows that $\operatorname{Ass}_{A} D=X$.

(c) This follows from (a) and (b).

Proof of Theorem 5.1. We consider the flat extension $\alpha: A \rightarrow \hat{A}$. Say $\hat{A}=Q /(\boldsymbol{f})$, where $(Q, \mathfrak{n})$ is a regular local ring and $f=f_{1}, \ldots, f_{c} \in \mathfrak{n}^{2}$ is a regular sequence.

(1) Consider $\mathscr{E}(N)=\bigoplus_{i \geq 0} \bigoplus_{n \geq 0} \operatorname{Ext}_{A}^{i}\left(M, N_{n}\right)$ as an $A$-module. By Theorem 1.1, $\mathscr{E}(N) \otimes \hat{A}$ is a finitely generated $B=\mathscr{R}(I \hat{A})\left[t_{1}, \ldots t_{c}\right]$-algebra. By Lemma 5.6 we get that $\operatorname{Ass}_{A} \mathscr{E}(N)$ is a finite set. Notice that

$$
\operatorname{Ass}_{A} \mathscr{E}(N)=\bigcup_{n \geq 0} \bigcup_{i \geq 0} \operatorname{Ass}_{A} \operatorname{Ext}_{A}^{i}\left(M, N_{n}\right)
$$

(2) Set $\mathscr{E}=\mathscr{E}(N)$. By Theorem 1.1 there exist $i_{0}$ and $n_{0}$ such that for all $i \geq i_{0}$ and $n \geq n_{0}$ we have

$$
\operatorname{Ass}_{\hat{A}} \mathscr{\mathscr { E }}_{2 i, n} \otimes \hat{A}=\operatorname{Ass}_{\hat{A}} \mathscr{\mathscr { E }}_{2 i_{0}, n_{0}} \otimes \hat{A}, \quad \operatorname{Ass}_{\hat{A}} \mathscr{\mathscr { E }}_{2 i+1, n} \otimes \hat{A}=\operatorname{Ass}_{\hat{A}} \mathscr{\mathscr { E }}_{2 i_{0}+1, n_{0}} \otimes \hat{A} .
$$

By Lemma 5.6(b) it follows that for all $i \geq i_{0}$ and $n \geq n_{0}$ we have

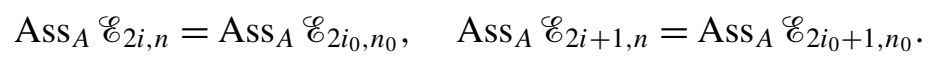

\section{Application II: Asymptotic associated primes - the geometric case}

Let $V$ be an affine or projective variety over an algebraically closed field $K$. Then $V$ is said to be a local complete intersection if all of its local rings are complete intersections. Let $A$ be the coordinate ring of $V$. In the affine case we have $A_{\mathfrak{p}}$ is a complete intersection for all $\mathfrak{p} \in \operatorname{Spec}(A)$. In the projective case we have $A_{(\mathfrak{p})}$ is a complete intersection for every $\mathfrak{p} \in \operatorname{Proj}(A)$. In this section we prove results analogous to Theorem 5.1 to coordinate rings of locally complete intersection varieties.

We first consider the affine case. In this case we prove the following general result. Recall a ring $R$ is regular (a complete intersection) if $R_{\mathfrak{p}}$ is regular (a complete intersection) for all $\mathfrak{p} \in \operatorname{Spec}(R)$.

Theorem 6.1. Let $Q$ be a regular ring of finite Krull dimension and let $\mathfrak{a}$ be an ideal in $Q$ with $A=Q / \mathfrak{a}$ a complete intersection. Let $M$ be a finitely generated A-module and let $I$ be an ideal in $A$. Let $N=\bigoplus_{n \geq 0} N_{n}$ be a finitely generated $\mathscr{R}(I)$-module. 
Then

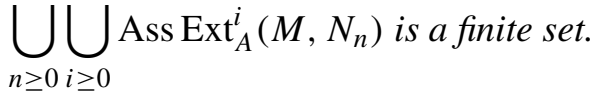

Furthermore there exist $i_{0}, n_{0}$ such that for all $i \geq i_{0}$ and $n \geq n_{0}$ we have

$$
\begin{aligned}
\operatorname{Ass}_{A} \operatorname{Ext}_{A}^{2 i}\left(M, N_{n}\right) & =\operatorname{Ass}_{A} \operatorname{Ext}_{A}^{2 i_{0}}\left(M, N_{n_{0}}\right), \\
\operatorname{Ass}_{A} \operatorname{Ext}_{A}^{2 i+1}\left(M, N_{n}\right) & =\operatorname{Ass}_{A} \operatorname{Ext}_{A}^{2 i_{0}+1}\left(M, N_{n_{0}}\right) .
\end{aligned}
$$

6.2. Before proving Theorem 6.1 we state the analogous result in the projective case. Let $\mathfrak{a}$ be a graded ideal in $Q=K\left[X_{0}, X_{1}, \ldots, X_{m}\right]$, where $\operatorname{deg} X_{i}=1$ for all $i$. Here $K$ is not necessarily algebraically closed. Set $A=Q / \mathfrak{a}$. We assume $A_{(\mathfrak{p})}$ is a complete intersection for every $\mathfrak{p} \in \operatorname{Proj} A$. Recall that if $U$ is the set of homogeneous elements in $A \backslash \mathfrak{p}$ then $A_{(\mathfrak{p})}$ is the degree-zero part of the graded ring $U^{-1} A$.

Let $\mathfrak{m}$ be the unique maximal homogeneous ideal of $A$. If $E$ is a graded $A$-module then note that all its associate primes are homogeneous prime ideals of $A$. Set

$$
{ }^{*} \operatorname{Ass}_{A}(E)=\operatorname{Ass}_{A}(E) \backslash\{\mathfrak{m}\}
$$

the relevant associate primes of $E$. In the projective case our main theorem is this:

Theorem 6.3. (Keep the hypotheses of Section 6.2; note that $\mathscr{R}(I)$ is a bigraded ring.) Let $M$ be a finitely generated graded A-module and let I be a homogeneous ideal in A. Let $N=\bigoplus_{n \geq 0} N_{n}$ be a finitely generated bigraded $\mathscr{R}(I)$-module (so each $N_{n}$ is a graded A-module). Then

$$
\begin{aligned}
& \bigcup{ }^{*} \operatorname{Ass} \operatorname{Ext}_{A}^{i}\left(M, N_{n}\right) \text { is a finite set. } \\
& n \geq 0 i \geq 0
\end{aligned}
$$

Furthermore there exist $i_{0}, n_{0}$ such that for all $i \geq i_{0}$ and $n \geq n_{0}$ we have

$$
\begin{aligned}
{ }^{*} \operatorname{Ass}_{A} \operatorname{Ext}_{A}^{2 i}\left(M, N_{n}\right) & ={ }^{*} \operatorname{Ass}_{A} \operatorname{Ext}_{A}^{2 i_{0}}\left(M, N_{n_{0}}\right), \\
{ }^{*} \operatorname{Ass}_{A} \operatorname{Ext}_{A}^{2 i+1}\left(M, N_{n}\right) & ={ }^{*} \operatorname{Ass}_{A} \operatorname{Ext}_{A}^{2 i_{0}+1}\left(M, N_{n_{0}}\right) .
\end{aligned}
$$

We now prove Theorems 6.1 and 6.3. We begin with the affine case. We need the following:

Lemma 6.4. Suppose $A=Q / \mathfrak{a}$, where $Q$ is a regular ring. Suppose for some $\mathfrak{p} \in \operatorname{Spec} A$ the ring $A_{\mathfrak{p}}$ is a complete intersection. Let $\mathfrak{q} \in \operatorname{Spec} Q$ with $\mathfrak{q} / \mathfrak{a}=\mathfrak{p}$. Then there exist $g \in Q \backslash \mathfrak{q}$ such that $\mathfrak{a} Q_{g}$ is generated by a $Q_{g}$-regular sequence. 
Proof. We have $A_{\mathfrak{p}}=Q_{\mathfrak{q}} / \mathfrak{a} Q_{\mathfrak{q}}$. Since $A_{\mathfrak{p}}$ is a complete intersection it follows from [Matsumura 1980, 21.2] that $\mathfrak{a} Q_{\mathfrak{q}}$ is generated by a regular sequence, say $f_{1}, \ldots, f_{c}$. We may assume $f_{i} \in \mathfrak{a}$ for all $i$.

Set

$$
E=\frac{\mathfrak{a}}{\left(f_{1}, \ldots, f_{c}\right)} \quad \text { and } \quad D_{i}=\frac{\left(f_{1}, \ldots, f_{i-1}\right): f_{i}}{\left(f_{1}, \ldots, f_{i-1}\right)} \quad \text { for } i=1, \ldots, c .
$$

Let

$$
L=E \oplus\left(\bigoplus_{i=1}^{c} D_{i}\right) .
$$

Then $L$ is a finitely generated $Q$-module and $L_{\mathfrak{q}}=0$. So there exists $g \in Q \backslash \mathfrak{q}$ such that $L_{g}=0$. In $Q_{g}$ note that $\mathfrak{a} Q_{g}=\left(f_{1}, \ldots, f_{c}\right) Q_{g}$. Also as $\left(f_{1}, \ldots, f_{c}\right) Q_{\mathfrak{q}} \neq Q_{\mathfrak{q}}$ we have that $\left(f_{1}, \ldots, f_{c}\right) Q_{g} \neq Q_{g}$. Since $\left(D_{i}\right)_{g}=0$ for $i=1, \ldots, c$ we get that $f_{1}, \ldots, f_{c}$ is a $Q_{g}$-regular sequence.

Proof of Theorem 6.1. Let $\mathfrak{p} \in \operatorname{Spec} A$. Then $A_{\mathfrak{p}}$ is a complete intersection. Let $\mathfrak{q} \in \operatorname{Spec} Q$ with $\mathfrak{q} / \mathfrak{a}=\mathfrak{p}$. Then by Lemma 6.4 there exist $g \in Q \backslash \mathfrak{q}$ such that $\mathfrak{a} Q_{g}$ is generated by a $Q$-regular sequence. Let $g_{\mathfrak{p}}$ be the image of $g$ in $A$.

For $x \in A$ let $D(x)=\{\mathfrak{P} \in \operatorname{Spec} A \mid x \notin \mathfrak{P}\}$. Then $D(x)$ is a basic open set in $\operatorname{Spec}(A)$. Note that $\mathfrak{p} \in D\left(g_{\mathfrak{p}}\right)$. Clearly

$$
\operatorname{Spec} A=\bigcup_{\mathfrak{p} \in \operatorname{Spec} A} D\left(g_{\mathfrak{p}}\right) .
$$

As $\operatorname{Spec} A$ is quasicompact we have

$$
\text { Spec } A=D\left(g_{\mathfrak{p}_{1}}\right) \cup \cdots \cup D\left(g_{\mathfrak{p}_{m}}\right) \text { for some } m \geq 1 \text {. }
$$

Set $g_{i}=g_{\mathfrak{p}_{i}}$. Note that for any $A$-module $E$ we have

$$
\operatorname{Ass}_{A} E=\bigcup_{i=1}^{m}\left(\operatorname{Ass}_{A_{g_{i}}} E_{g_{i}}\right) \cap A,
$$

and that $\mathscr{E}(N)_{g}=\mathscr{E}\left(N_{g}\right)$. Thus it suffices to prove the result for $A_{g_{i}}$ for each $i$.

For each $i=1, \ldots, m$ we have that

$$
A_{g_{i}}=\frac{Q_{i} \text { a regular ring of finite Krull dimension }}{\text { regular sequence in } Q_{i}} .
$$

As $Q_{i}$ is a regular ring of finite Krull dimension we get that $\operatorname{projdim}_{Q_{i}} M_{g_{i}}$ is finite. So we can apply Theorem 5.2 to get the result.

To prove Theorem 6.3 we need a few preliminaries. Recall that a $\mathbb{Z}$-graded ring $S=\bigoplus_{n \in \mathbb{Z}} S_{n}$ is said to be $*$-local if it has a unique proper maximal homogeneous ideal $\mathfrak{P}$. Note that $\mathfrak{P}$ is a prime ideal in $S$ but not necessarily a maximal ideal in $S$. The functor $-\otimes S_{\mathfrak{P}}$ from the category of graded $S$-modules to the category of 
$S_{\mathfrak{P}}$-modules is faithfully exact, by [Bruns and Herzog 1993, 1.5.15]. The following result is well known and can be easily proved using the same reference.

Lemma 6.5. Let $S=\bigoplus_{n \in \mathbb{Z}} S_{n}$ be a $*$-local Cohen-Macaulay ring with unique maximal homogeneous ideal $\mathfrak{P}$. Let $\mathfrak{a}$ be a homogeneous ideal in $S$. If $\mathfrak{a}_{\mathfrak{P}}$ is generated by a regular sequence then $\mathfrak{a}$ is generated by a regular sequence of homogeneous elements. Furthermore if $\mathfrak{b}=\left\{x_{\alpha} \mid \alpha \in \Delta\right\}$ is a generating set of $\mathfrak{a}$ consisting of homogeneous elements then we may choose $\boldsymbol{x}=x_{1}, \ldots, x_{c} \in \mathscr{C}$ with $\mathfrak{a}=(\boldsymbol{x})$ and $\boldsymbol{x}$ is an $S$-regular sequence.

To prove Theorem 6.3 we need the following analogue of Lemma 6.4.

Lemma 6.6. Suppose $A=Q / \mathfrak{a}$, where $Q=K\left[X_{0}, \ldots, X_{n}\right]$ is graded with $\operatorname{deg} X_{i}=$ 1 for all $i$ and $\mathfrak{a}$ is a homogeneous ideal in $Q$. Suppose for some $\mathfrak{p} \in \operatorname{Proj} A$ the ring $A_{(\mathfrak{p})}$ is a complete intersection. Let $\mathfrak{q} \in \operatorname{Proj} Q$ with $\mathfrak{q} / \mathfrak{a}=\mathfrak{p}$. Then there exist homogeneous $g \in Q \backslash \mathfrak{q}$ such that $\mathfrak{a} Q_{g}$ is generated by a $Q_{g}$-regular sequence.

Proof. Set

$$
\begin{aligned}
U & =\{h \in A \mid h \text { homogeneous and } h \notin \mathfrak{p}\}, \\
W & =\{h \in Q \mid h \text { homogeneous and } h \notin \mathfrak{q}\} .
\end{aligned}
$$

Then $U^{-1} A=W^{-1} Q / W^{-1} \mathfrak{a}$. Also note that some $X_{i} \notin \mathfrak{q}$. It follows that

$$
U^{-1} A \cong A_{(\mathfrak{p})}\left[t, t^{-1}\right] \quad \text { and } \quad W^{-1} Q \cong Q_{(\mathfrak{q})}\left[t, t^{-1}\right] .
$$

Claim. $U^{-1} A$ is a complete intersection.

To see this, first observe that as $Q_{\mathfrak{q}}$ is a localization of $W^{-1} Q$ we have a flat map $Q_{(\mathfrak{q})} \rightarrow Q_{\mathfrak{q}}$ of local rings. As $Q_{\mathfrak{q}}$ is regular we have that $Q_{(\mathfrak{q})}$ is regular (see [Matsumura 1980, 23.7]). Notice that $A_{(\mathfrak{p})}$ is a quotient of a regular local ring $Q_{(\mathfrak{q})}$. So by [Bruns and Herzog 1993, 2.3.6], we have that $A_{(\mathfrak{p})}[t]$ is a complete intersection. As $U^{-1} A$ is a localization of $A_{(\mathfrak{p})}[t]$, it is also a complete intersection.

By Lemma 6.5 we have that $W^{-1} \mathfrak{a}$ is generated by a regular sequence $\boldsymbol{x}=$ $x_{1}, \ldots, x_{c}$ with $\boldsymbol{x} \in \mathfrak{a}$ homogeneous. Set

$$
E=\frac{\mathfrak{a}}{\left(x_{1}, \ldots, x_{c}\right)} \quad \text { and } \quad D_{i}=\frac{\left(x_{1}, \ldots, x_{i-1}\right): x_{i}}{\left(x_{1}, \ldots, x_{i-1}\right)} \quad \text { for } i=1, \ldots, c .
$$

Set

$$
L=E \oplus\left(\bigoplus_{i=1}^{c} D_{i}\right)
$$

We have $W^{-1} L=0$. Also, $L$ is a finitely generated $Q$-module. So there exist $g \in W$ with $L_{g}=0$. In $Q_{g}$ note that $\mathfrak{a} Q_{g}=\left(x_{1}, \ldots, x_{c}\right) Q_{g}$. Also, as $\left(x_{1}, \ldots, x_{c}\right) W^{-1} Q \neq$ $W^{-1} Q$ we have that $\left(x_{1}, \ldots, x_{c}\right) Q_{g} \neq Q_{g}$. Since $\left(D_{i}\right)_{g}=0$ for $i=1, \ldots, c$ we get that $x_{1}, \ldots, x_{c}$ is a $Q_{g}$-regular sequence. 
The proof of Theorem 6.3 is similar to that of Theorem 6.1, so we just sketch it. Sketch of proof of Theorem 6.3. We use Lemma 6.6 and an argument analogous to the one used Theorem 6.1 to obtain

$$
\text { Proj } A={ }^{*} D\left(g_{1}\right) \cup \cdots \cup{ }^{*} D\left(g_{r}\right) \text { for some } r \geq 1,
$$

for some homogeneous $g_{i} \in A$ and $A_{g_{i}}=Q_{i} / \mathfrak{a}_{i}$, where $Q_{i}$ is regular of finite Krull dimension and $\mathfrak{a}_{i}$ is generated by a regular sequence. Note that for $x$ homogeneous, ${ }^{*} D(x)=\{\mathfrak{P} \in \operatorname{Proj} A \mid x \notin \mathfrak{P}\}$.

Let $E$ be a graded $A$-module. Note that

$$
{ }^{*} \operatorname{Ass}_{A} E=\bigcup_{i=1}^{r}\left(\operatorname{Ass}_{A_{g_{i}}} E_{g_{i}}\right) \cap A .
$$

The result now follows by applying Theorem 5.2 to each $A_{g_{i}}=Q_{i} / \mathfrak{a}_{i}$.

\section{Application III: Support varieties}

Let $(A, \mathfrak{m})$ be a local complete intersection of codimension $c$. Let $M, N$ be two finitely generated $A$-modules. Define

$$
\operatorname{cx}_{A}(M, N)=\inf \left\{b \in \mathbb{N} \mid \varlimsup_{n \rightarrow \infty} \frac{\mu\left(\operatorname{Ext}_{A}^{n}(M, N)\right)}{n^{b-1}}<\infty\right\} .
$$

In this section we prove the following theorem:

Theorem 7.1. Let $(A, \mathfrak{m})$ be a local complete intersection, $M, N$ two finitely generated A-modules and let I be a proper ideal in A. Then

$$
\mathrm{cx}_{A}\left(M, I^{j} N\right) \text { is constant for all } j \gg 0 \text {. }
$$

7.2. Reduction to the case when $A$ is complete and the residue field of $A$ is algebraically closed.

7.3. Suppose $A^{\prime}$ is a flat local extension of $A$ such that $\mathfrak{m}^{\prime}=\mathfrak{m} A^{\prime}$ is the maximal ideal of $A^{\prime}$. If $E$ is an $A$-module then set $E^{\prime}=E \otimes_{A} A^{\prime}$. Notice that $I^{\prime} \cong I A^{\prime}$; we consider it as an ideal in $A^{\prime}$. By [Avramov 1998, 7.4.3], $A^{\prime}$ is also a complete intersection. It can be easily checked that

$$
\operatorname{cx}_{A^{\prime}}\left(M^{\prime},\left(I^{\prime}\right)^{j} N^{\prime}\right)=\operatorname{cx}_{A}\left(M, I^{j} N\right) \text { for all } n \geq 0 .
$$

We now do our reduction in two steps.

By [Bourbaki 1983, Chapitre 9, appendice, corollaire du théoréme 1, p. IX.41], there exists a flat local extension $A \subseteq \widetilde{A}$ such that $\widetilde{\mathfrak{m}}=\mathfrak{m} \widetilde{A}$ is the maximal ideal of $\tilde{A}$ and the residue field $\tilde{k}$ of $\tilde{A}$ is an algebraically closed extension of $k$. By 
Section 7.3 it follows that we may assume $k$ to be algebraically closed. We now complete $A$. Note that $\hat{A}$ is a flat extension of $A$ which satisfies Section 7.3.

Thus we may assume that our local complete intersection $A$

(1) is complete. So $A=Q /\left(f_{1}, \ldots, f_{c}\right)$, where $(Q, \mathfrak{n})$ is regular local and $f_{1}, \ldots, f_{c} \in \mathfrak{n}^{2}$ is a regular sequence.

(2) has an algebraically closed residue field $k$.

Of course there exist many $Q$ and $f_{1}, \ldots, f_{c}$ of the type as indicated above. We simply fix one such representation of $A$.

7.4. Let $U, V$ be two finitely generated $A$-modules.

Let $\operatorname{Ext}^{*}(U, V)=\bigoplus_{n \geq 0} \operatorname{Ext}_{A}^{n}(U, V)$ be the total ext module of $U$ and $V$. We consider it as a (finitely generated) module over the ring of cohomological operators $A\left[t_{1}, \ldots, t_{c}\right]$. Since $\operatorname{projdim}_{Q} U$ is finite $\operatorname{Ext}^{*}(U, V)$ is a finitely generated $A\left[t_{1}, \ldots, t_{c}\right]$-module.

7.5. Let $\mathcal{C}(U, V)=\operatorname{Ext}^{*}(U, V) \otimes_{A} k$. Clearly $\mathcal{C}(U, V)$ is a finitely generated $T=k\left[t_{1}, \ldots, t_{c}\right]$-module. (Here the degree of $t_{i}$ is 2 for each $i=1, \ldots, c$ ). Set

$$
\mathfrak{a}(U, V)=\operatorname{ann}_{T} \mathcal{C}(U, V) .
$$

Notice that $\mathfrak{a}(U, V)$ is a homogeneous ideal.

7.6. We now forget the grading of $T$ and consider the affine space $A^{c}(k)$. Let

$$
\mathscr{V}(U, V)=\mathscr{V}(\mathfrak{a}(U, V)) \subseteq \mathbb{A}^{c}(k) .
$$

Since $\mathfrak{a}(U, V)$ is a graded ideal we get that $\mathscr{V}(U, V)$ is a cone.

7.7. By [Avramov and Buchweitz 2000, 2.4] we get that

$$
\operatorname{dim} \mathscr{V}(U, V)=\operatorname{cx}_{A}(M, N) .
$$

Lemma 7.8. If I is an ideal in $A$ then there exists $j_{0} \geq 0$ such that

$$
\mathscr{V}\left(U, I^{j} V\right)=\mathscr{V}\left(U, I^{j_{0}} V\right) \text { for all } j \geq j_{0} .
$$

Proof of Theorem 7.1 assuming the lemma. By 7.3 we may assume that $A$ is complete and has an algebraically closed residue field. The result now follows from 7.7 and Lemma 7.8 .

7.9. Let $N=\bigoplus_{n \geq 0} I^{n} V$. Set $\mathscr{E}(N)=\bigoplus_{n \geq 0} \bigoplus_{i \geq 0} \operatorname{Ext}_{A}^{i}\left(U, I^{n} V\right)$. Set $\mathcal{C}(N)=$ $\mathscr{E}(N) \otimes_{A} k$. By Theorem $1.1, \mathscr{E}(N)$ is a finitely generated $\mathscr{S}=\mathscr{R}(I)\left[t_{1}, \ldots, t_{c}\right]$ module. It follows that $\mathcal{C}(N)$ is a finitely generated, bigraded, $G=F(I)\left[t_{1}, \ldots, t_{c}\right]$ module. Recall that $F(I)$, the fiber cone of $I$, is a finitely generated $k$-algebra. 
So we may as well consider $\mathcal{C}(N)$ as a bigraded $R=k\left[X_{1}, \ldots, X_{m}, t_{1}, \ldots t_{c}\right]$ module (of course here $X_{1}, \ldots, X_{m}$ are variables). Furthermore deg $X_{l}=(1,0)$ for $l=1, \ldots m$ and $\operatorname{deg} t_{s}=(0,2)$ for $s=1, \ldots, c$. Set $T=k\left[t_{1}, \ldots, t_{c}\right]$.

7.10. Advantages of coarsening the grading on $\mathrm{C}(N)$. By forgetting the degree on the $t_{i}$ we may consider $R=T\left[X_{1}, \ldots, X_{m}\right]$. Notice that

$$
\mathcal{C}(N)=\bigoplus_{n \geq 0} \mathcal{C}\left(U, I^{n} V\right)
$$

as a graded $R$-module.

Proof of Lemma 7.8. We make the constructions as in Section 7.10. So $\mathrm{C}(N)$ is a finitely generated graded $R=T\left[X_{1}, \ldots, X_{m}\right]$-module. Notice that $R$ is $\mathbb{N}$-standard graded. So there exists $j_{0}$ such that

$$
\operatorname{ann}_{T} \mathrm{C}(N)_{j}=\operatorname{ann}_{T} \mathrm{C}(N)_{j_{0}} \text { for all } j \geq 0 .
$$

The results follows.

Question 7.11 (With hypotheses as in Theorem 7.1).

$$
\text { Is } \operatorname{cx}_{A}\left(M, N / I^{j} N\right) \text { constant for all } j \gg 0 \text { ? }
$$

\section{Acknowledgements}

I thank Professor L. L. Avramov and Professor J. Herzog for many discussions regarding this paper. I also thank the referee for many pertinent comments.

\section{References}

[Avramov 1989] L. L. Avramov, "Modules of finite virtual projective dimension”, Invent. Math. 96:1 (1989), 71-101. MR 90g:13027 Zbl 0677.13004

[Avramov 1998] L. L. Avramov, "Infinite free resolutions", pp. 1-118 in Six lectures on commutative algebra (Bellaterra, 1996), edited by J. Elias et al., Progr. Math. 166, Birkhäuser, Basel, 1998. MR 99m:13022 Zbl 0934.13008

[Avramov and Buchweitz 2000] L. L. Avramov and R.-O. Buchweitz, "Support varieties and cohomology over complete intersections", Invent. Math. 142:2 (2000), 285-318. MR 2001j:13017 Zbl 0999.13008

[Avramov et al. 1997] L. L. Avramov, V. N. Gasharov, and I. V. Peeva, "Complete intersection dimension”, Inst. Hautes Études Sci. Publ. Math. 86 (1997), 67-114. MR 99c:13033 Zbl 0918.13008

[Bourbaki 1983] N. Bourbaki, Algèbre commutative, chapitres 8 et 9, Masson, Paris, 1983. Reprinted Springer, Berlin, 2006. MR 86j:13001 Zbl 0579.13001

[Brodmann 1979] M. Brodmann, "Asymptotic stability of $\operatorname{Ass}\left(M / I^{n} M\right)$ ", Proc. Amer. Math. Soc. 74:1 (1979), 16-18. MR 80c:13012 Zbl 0395.13008

[Bruns and Herzog 1993] W. Bruns and J. Herzog, Cohen-Macaulay rings, Cambridge Studies in Advanced Mathematics 39, Cambridge University Press, 1993. MR 95h:13020 Zbl 0788.13005 
[Eisenbud 1980] D. Eisenbud, "Homological algebra on a complete intersection, with an application to group representations", Trans. Amer. Math. Soc. 260:1 (1980), 35-64. MR 82d:13013 Zbl 0444.13006

[Gulliksen 1974] T. H. Gulliksen, "A change of ring theorem with applications to Poincaré series and intersection multiplicity”, Math. Scand. 34 (1974), 167-183. MR 51 \#487 Zbl 0292.13009

[Heitmann and Jorgensen 2012] R. C. Heitmann and D. A. Jorgensen, "Are complete intersections complete intersections?”, J. Algebra 371 (2012), 276-299. MR 2975397 Zbl 06166659

[Katz and West 2004] D. Katz and E. West, "A linear function associated to asymptotic prime divisors", Proc. Amer. Math. Soc. 132:6 (2004), 1589-1597. MR 2005d:13024 Zbl 1057.13002

[Matsumura 1980] H. Matsumura, Commutative ring theory, Kyoritsu Shuppan, Tokyo, 1980. In Japanese; translated in Cambridge Studies in Advanced Mathematics 8, 2nd ed., Cambridge University Press, 1989. MR 90i:13001 Zbl 0666.13002

[Melkersson and Schenzel 1993] L. Melkersson and P. Schenzel, "Asymptotic prime ideals related to derived functors", Proc. Amer. Math. Soc. 117:4 (1993), 935-938. MR 93e:13027 Zbl 0780.13003

[Puthenpurakal 2005] T. J. Puthenpurakal, "The Hilbert function of a maximal Cohen-Macaulay module”, Math. Z. 251:3 (2005), 551-573. MR 2006i:13022 Zbl 1158.13306

[Singh 2000] A. K. Singh, " $p$-torsion elements in local cohomology modules", Math. Res. Lett. 7:2-3 (2000), 165-176. MR 2001g:13039 Zbl 0965.13013

[Vasconcelos 1998] W. V. Vasconcelos, "Cohomological degrees of graded modules", pp. 345-392 in Six lectures on commutative algebra (Bellaterra, 1996), edited by J. Elias et al., Progr. Math. 166, Birkhäuser, Basel, 1998. MR 99j:13012 Zbl 0931.13016

[West 2004] E. West, "Primes associated to multigraded modules", J. Algebra 271:2 (2004), 427-453. MR 2004m:13004 Zbl 1036.13002

Received September 18, 2008. Revised April 11, 2013.

TONY J. PUTHENPURAKAL

DEPARTMENT OF MATHEMATICS

INDIAN INSTITUTE OF TECHNOLOGY BOMBAY

POWAI

MUMBAI 400076

INDIA

tputhen@math.iitb.ac.in 


\title{
PACIFIC JOURNAL OF MATHEMATICS
}

\author{
msp.org/pjm
}

Founded in 1951 by E. F. Beckenbach (1906-1982) and F. Wolf (1904-1989)

\section{EDITORS}

V. S. Varadarajan (Managing Editor)

Department of Mathematics

University of California

Los Angeles, CA 90095-1555

pacific@math.ucla.edu

Paul Balmer

Department of Mathematics

University of California

Los Angeles, CA 90095-1555

balmer@math.ucla.edu

Daryl Cooper

Department of Mathematics

University of California

Santa Barbara, CA 93106-3080 cooper@math.ucsb.edu

Jiang-Hua Lu

Department of Mathematics

The University of Hong Kong

Pokfulam Rd., Hong Kong jhlu@maths.hku.hk
Don Blasius

Department of Mathematics University of California

Los Angeles, CA 90095-1555

blasius@math.ucla.edu

Robert Finn

Department of Mathematics Stanford University

Stanford, CA 94305-2125

finn@math.stanford.edu

Sorin Popa

Department of Mathematics

University of California

Los Angeles, CA 90095-1555 popa@math.ucla.edu

Paul Yang

Department of Mathematics Princeton University

Princeton NJ 08544-1000

yang@math.princeton.edu

\section{PRODUCTION}

Silvio Levy, Scientific Editor, production@msp.org

\section{SUPPORTING INSTITUTIONS}

ACADEMIA SINICA, TAIPEI

CALIFORNIA INST. OF TECHNOLOGY

INST. DE MATEMÁTICA PURA E APLICADA

KEIO UNIVERSITY

MATH. SCIENCES RESEARCH INSTITUTE

NEW MEXICO STATE UNIV.

OREGON STATE UNIV.

\author{
STANFORD UNIVERSITY \\ UNIV. OF BRITISH COLUMBIA \\ UNIV. OF CALIFORNIA, BERKELEY \\ UNIV. OF CALIFORNIA, DAVIS \\ UNIV. OF CALIFORNIA, LOS ANGELES \\ UNIV. OF CALIFORNIA, RIVERSIDE \\ UNIV. OF CALIFORNIA, SAN DIEGO \\ UNIV. OF CALIF., SANTA BARBARA
}

\author{
Vyjayanthi Chari \\ Department of Mathematics \\ University of California \\ Riverside, CA 92521-0135 \\ chari@math.ucr.edu \\ Kefeng Liu \\ Department of Mathematics \\ University of California \\ Los Angeles, CA 90095-1555 \\ liu@math.ucla.edu \\ Jie Qing \\ Department of Mathematics \\ University of California \\ Santa Cruz, CA 95064 \\ qing@cats.ucsc.edu
}

These supporting institutions contribute to the cost of publication of this Journal, but they are not owners or publishers and have no responsibility for its contents or policies.

See inside back cover or msp.org/pjm for submission instructions.

The subscription price for 2013 is US \$400/year for the electronic version, and \$485/year for print and electronic.

Subscriptions, requests for back issues and changes of subscribers address should be sent to Pacific Journal of Mathematics, P.O. Box 4163, Berkeley, CA 94704-0163, U.S.A. The Pacific Journal of Mathematics is indexed by Mathematical Reviews, Zentralblatt MATH, PASCAL CNRS Index, Referativnyi Zhurnal, Current Mathematical Publications and Web of Knowledge (Science Citation Index).

The Pacific Journal of Mathematics (ISSN 0030-8730) at the University of California, c/o Department of Mathematics, 798 Evans Hall \#3840, Berkeley, CA 94720-3840, is published twelve times a year. Periodical rate postage paid at Berkeley, CA 94704, and additional mailing offices. POSTMASTER: send address changes to Pacific Journal of Mathematics, P.O. Box 4163, Berkeley, CA 94704-0163.

PJM peer review and production are managed by EditFLOW ${ }^{\circledR}$ from Mathematical Sciences Publishers.

\section{PUBLISHED BY}

mathematical sciences publishers

nonprofit scientific publishing

http://msp.org/

(C) 2013 Mathematical Sciences Publishers 


\section{PACIFIC JOURNAL OF MATHEMATICS}

Volume $266 \quad$ No. $2 \quad$ December 2013

Rate of attraction for a semilinear wave equation with variable coefficients and

critical nonlinearities

FÁGNER DiAs ARARUNA and FLANK DAVID MORAIS BEZERRA

The Brin-Thompson groups $s V$ are of type $\mathrm{F}_{\infty}$

Martin G. Fluch, Marco Marschler, Stefan Witzel and

MATTHEW C. B. ZAREMSKY

Ideal decompositions of a ternary ring of operators with predual

MASAYOSHI KANEDA

A study of real hypersurfaces with Ricci operators in 2-dimensional complex space 305 forms

Dong Ho Lim, WoOn Ha SoHn and Hyunjung Song

On commensurability of fibrations on a hyperbolic 3-manifold

HideTOSHI MASAI

Multiplicative Dirac structures

CRISTIÁN ORTIZ

On the finite generation of a family of Ext modules

TONY J. PuthenPuRAKAL

Index formulae for Stark units and their solutions

XAVIER-FranÇOIS ROBLOT

The short time asymptotics of Nash entropy

GUOYI XU

Several splitting criteria for vector bundles and reflexive sheaves

STEPHEN S.-T. YAU and FEI YE

The minimal volume orientable hyperbolic 3-manifold with 4 cusps

KEN'ICHI YOSHIDA

On the Witten rigidity theorem for string ${ }^{c}$ manifolds

JIANQING YU and BO LIU 